\title{
Desequilíbrio entre a oferta e a demanda brasileira por pescados em 2002/2003 e 2008/2009
}

\author{
Brazilian fishery supply and demand inequality in 2002/2003 and 2008/2009
}

\author{
Daniel Yokoyama Sonoda1*; Ricardo Shirota ${ }^{1}$; João Donato Scorvo Filho3; José \\ Eurico Possebon Cyrino ${ }^{4}$
}

\footnotetext{
1 PECEGE - Doutor em Ciências (Economia Aplicada) - Rua Alexandre Herculano, 120, sala T4 - Vila Monteira - CEP 13418-4450 - Piracicaba (SP), Brasil

${ }^{2}$ ESALQ/USP - Prof. Doutor em Economia Rural - Av. Pádua Dias 11, Departamento de Economia Administração e Sociologia - Bairro Agronomia - CEP 13418-900 - Piracicaba (SP), Brasil

${ }^{3}$ APTA - Doutor em Aquicultura - Estrada Municipal Monte Alegre - Pinhalzinho, km 3, Polo Regional Leste Paulista Caixa Postal 01 - CEP 13910-000 - Monte Alegre do Sul (SP), Brasil

${ }^{4}$ ESALQ/USP - Prof. Doutor em Fisheries and Allied Aquacultures - Av. Pádua Dias 11, Departamento de Zootecnia Bairro Agronomia - CEP 13418-900 - Piracicaba (SP), Brasil
}

\section{Resumo}

A produção nacional de pescados entre 2003 e 2009 aumentou em 250,54 mil t, o déficit da balança comercial bruta de pescados aumentou em 285,48 mil t, porém, não se constatou elevação do consumo domiciliar bruto de pescados no mesmo período. Este trabalho propõe um método de análise baseado no equilíbrio entre a oferta e a demanda agregada de pescados nos períodos de 2002-2003 e 2008-2009 para verificar se existe a coerência entre o crescimento da oferta e a destinação dos pescados (consumo domiciliar e fora de casa). Os cálculos indicam que enquanto os dados oficiais apontam um crescimento do consumo per capita aparente de 2 $\mathrm{kg} / \mathrm{hab}$.ano, os dados corrigidos apresentam um crescimento mais modesto (em torno de 0,6 $\mathrm{kg} / \mathrm{hab}$.ano) entre os anos de 2002-2003 e 2008-2009.

Palavras-chave: equilíbrio, balança comercial, aquicultura, pesca

\section{Abstract}

Brazilian fish production between 2003 and 2009 increased by $250.54 t$, gross fish trade deficit increased at 285.48t, but the household consumption of fish was flat in the same period. This paper proposes an analysis of fish aggregate supply and demand balance in the periods of 2002-2003 and 2008-2009 to check the consistency between supply growth and allocation of fish (household consumption and eating out). While official data indicate $2 \mathrm{~kg} / \mathrm{hab}$.year increases in apparent per capita fishery consumption, the corrected data shows $0.6 \mathrm{~kg} / \mathrm{hab}$.year increase between 2002-2003 and 2008-2009.

Key-words: equilibrium, trade, aquaculture, fisheries

\footnotetext{
* Autor correspondente < dysonoda@pecege.com> Enviado: 04 ago. 2014

Aprovado: 28 nov. 2014
} 


\section{Introdução}

Desde a década de 1990, praticamente todo crescimento produção mundial de pescados vem ocorrendo em função da expansão da aquicultura. No Brasil, apesar das dificuldades legais: dificuldade para obtenção de licenciamento ambiental; da excessiva carga tributária; o difícil acesso ao crédito; infraestrutura deficitária e falta de um programa governamental para a extensão e assistência técnica aquícola; a aquicultura, ainda responde por boa parte do crescimento da produção nacional de pescados.

Em 2009, a produção nacional proveniente exclusivamente da aquicultura, foi de 416 mil toneladas, o que representa $33 \%$ do volume total de pescados e $0,75 \%$ da produção mundial (FAO, 2010). Na China, país líder nesse ranking, cuja produção é de 60 milhões de toneladas, 75\% é proveniente da aquicultura. Essa diferença, associada à enorme disponibilidade de águas indica, o quanto o Brasil ainda pode crescer neste setor.

Segundo informações do Ministério da Pesca e Aquicultura (MPA, 2012) o consumo per capita aparente de pescado no Brasil foi de $9,75 \mathrm{~kg} / \mathrm{ano}$, em 2010, muito abaixo do valor mundial, que é superior a $17 \mathrm{~kg} / \mathrm{hab}$.ano. Este consumo foi, aproximadamente, $8 \%$ maior que o ano anterior e apresenta um crescimento acumulado de $44 \%$ desde 2001, entretanto, ainda está aquém da recomendação da Organização Mundial da Saúde - OMS, que indica um consumo de 12 kg/hab.ano (Sidonio et al., 2012).

O aumento deste consumo tem sido possível devido às importações, uma vez que a produção de pescado no Brasil, apesar do aumento da produção pela aquicultura, se mantém entre 1 milhão e 1,2 milhões de toneladas na última década.

Apesar dos dados oficiais apontarem um crescimento do consumo aparente de pescado nacional, a destinação por segmento (domicílio e consumo fora de casa ${ }^{2}$ ) apresentou-se praticamente estável. A primeira vista, existem possíveis divergências entre os dados oficiais da oferta e da demanda de pescados. Enquanto a produção de pescados de 2003 para 2009 aumentou em 250,54 mil t (MPA, 2012) e o déficit da balança comercial aumentou em 285,48 mil t (MDIC, 2012), o consumo domiciliar de acordo com dados da Pesquisa de Orçamento Familiar- POF manteve-se praticamente constante no mesmo período (IBGE, 2004 e 2011).

A oferta brasileira de pescados pode ser dividida em dois grandes grupos: a produção interna e a balança comercial de pescados (importações menos as

\footnotetext{
2 bares, restaurantes, cozinhas industriais, pesque-pagues
} 
exportações). A produção de pescados por sua vez, pode ser dividida em dois grupos: captura e aquicultura.

Em 2011, as estatísticas de produção nacional de pescado continuam apresentando um crescimento de 13,2\% em relação a 2010 (1.432 mil t e 1.265 mil t, respectivamente). A aquicultura, como principal fonte de produção de pescado nacional, responsável por $43,9 \%$ da produção total de pescado, seguida pela pesca extrativa marinha com $38,7 \%$ e a pesca extrativa continental $17,4 \%$ (MPA, 2013).

No caso da demanda por pescados, estudos mais recentes conduzidos pelo Centro para serviços de informações e assessoramento sobre a comercialização de produtos pesqueiros na América Latina e Caribe - Infopesca (Neiva et al., 2010; Barroso; Wiefels, 2010; Borges, 2010; Gandra, 2010) estimam o consumo de pescados em quatro grandes capitais brasileiras: São Paulo, Rio de Janeiro, Brasília e Manaus, para o ano de 2009.

O consumo total de pescado inteiro estimado para estas quatro capitais é de 613 mil $t$, sendo o consumo fora de casa responsável por aproximadamente 235 t e o consumo per capita médio de 20,39 kg/hab.ano. Ao confrontarmos os resultados desta pesquisa com os resultados da POF 2008-2009, percebe-se que, o consumo domiciliar destas quatro capitais representou quase metade do consumo domiciliar do Brasil (775 mil t em 2002-2003 e 772 mil t em 2008-2009, segundo a POF (IBGE, 2004 e 2011). Cabe ressaltar que apesar dos dados da POF não trazerem a equivalência em pescado inteiro, grande parte da aquisição de pescados para o consumo domiciliar ainda é feita desta forma (com cabeça, escama e vísceras).

Praticamente todo o crescimento da produção nacional de pescados ocorreu por meio da aquicultura. Embora não seja possível discriminar pelos dados da POF se o produto consumido foi oriundo da pesca ou da aquicultura, observa-se que houve um incremento de $21 \%$ no consumo domiciliar de quatro importantes espécies provenientes, principalmente, desta atividade zootécnica3: tilápia, carpa, camarão e moluscos bivalves entre os períodos estudados.

Os dados levantados indicam o aumento de produção e da importação de pescados entre os períodos analisados, porém o consumo domiciliar apresentou uma leve retração. Os dados de oferta e a demanda incluem pescados inteiros e pescados com diferentes graus de processamento, o que faz com que sua comparação possa ficar comprometida. Assim sendo, é preciso fazer uma correção para que se obtenha uma

\footnotetext{
${ }^{3}$ Apesar dos peixes redondos e bagres já serem produzidos pela piscicultura, não foram utilizados, pois sua captura ainda é bastante significativa nas regiões Norte e Centro Oeste, situação que poderia comprometer os resultados da análise.
} 
mesma base de comparação em massa. Imposta a condição de equilíbrio, pretende-se verificar de que forma o crescimento da oferta foi absorvido demanda.

Levantar este tipo de questionamento é importante para o direcionamento de políticas públicas de incentivo para a atividade, para auxiliar os empresários do setor a traçar suas metas sobre o destino de sua produção e também contribui para o aprimoramento da metodologia de coleta de dados estatísticos relacionados à pesca.

Este trabalho propõe um método de análise baseado no equilíbrio entre a oferta e a demanda agregada de pescados nos períodos de 2002-2003 e 2008-2009 para verificar se existe coerência entre o crescimento da oferta e a destinação dos pescados.

\section{Materiais e Métodos}

Os dados utilizados para a oferta de pescado foram disponibilizados pelo Ministério da Pesca e Aquicultura (MPA, 2012), por meio de seus Boletins Estatísticos da Pesca e Aquicultura e pelo Ministério do Desenvolvimento, Indústria e Comércio Exterior (MDIC, 2012) pelo sistema Aliceweb2. As informações da demanda de pescado foram obtidas a partir dos micros dados da Pesquisa de Orçamento Familiar (POF) do Instituto Brasileiro de Geografia e Estatística (IBGE, 2004 e IBGE, 2011). A POF é uma pesquisa executada com um intervalo de tempo maior do que as Estatísticas da Pesca e da Aquicultura que são anuais, portanto, as analises foram feitas seguindo o calendário da POF, junho de 2002 a junho 2003 e maio de 2008 a maio de 2009.

Devido à disponibilidade de dados, para análise da produção foram considerados pescados inteiros, já para a importação e exportação consideram tanto pescados inteiros quanto processados. A demanda, por sua vez, também considera pescados inteiros e processados. Ao se considerar pescados processados, existem partes que são descartadas dependendo do tipo de processamento que proporcionam perdas de massa em relação aos pescados inteiros. Portanto, procedeu-se a um processo de equivalência entre tipo de corte e pescado inteiro, uniformizando a unidade de medida. Ao se considerar as equivalências de pescados inteiros tanto na oferta quanto na demanda, minimizam-se as divergências de pesos. Os critérios para a conversão de pescados processados para inteiros foram os mesmos utilizados pelo Ministério da Pesca e Aquicultura (MPA, 2012).

Para a definição da oferta nacional de pescados foi considerado o consumo aparente de pescados, segundo a fórmula:

$$
\mathrm{O}=\mathrm{P}+\mathrm{I}_{\mathrm{e}}-\mathrm{E}_{\mathrm{e}}
$$




$$
\begin{aligned}
\mathrm{I}_{\mathrm{e}} & =\mathrm{I} \times \mathrm{f}_{\mathrm{I}} \\
\mathrm{E}_{\mathrm{e}} & =\mathrm{E} \times \mathrm{f}_{\mathrm{E}}
\end{aligned}
$$

Onde, $\mathrm{O}=$ Oferta de Pescado Inteiro (t); $\mathrm{P}$ = Produção Nacional de Pescados ( $\mathrm{t}$ ); le = Importação Equivalente de Pescado Inteiro (t); I = Importação Bruta de Pescados $(\mathrm{t}) ; \mathrm{fl}=$ Fator de correção para equivalente pescado inteiro importado $(1<\mathrm{fi}<4)$; Ee = Exportação Equivalente de Pescado Inteiro (t); E = Exportação Bruta de Pescado Inteiro $(\mathrm{t}) ; \mathrm{fE}=$ Fator de correção para equivalente pescado inteiro exportado $(1<\mathrm{fe}<4)$.

O ajuste dos dados da oferta de pescados para o período da POF foi feito através da média simples entre os anos envolvidos:

$$
\frac{\mathrm{O}_{2002}+\mathrm{O}_{2003}}{2} \text { e } \frac{\mathrm{O}_{2008}+\mathrm{O}_{2009}}{2}
$$

Para calcular a demanda de pescados foram utilizados os micro dados da POF 2002-2003 e da POF 2008-2009. Em 2002-2003 foram pesquisados 48.470 domicílios e em 2008-2009, 55.970 domicílios no Brasil. Os registros dos micros dados da POF que foram extraídos, combinados, filtrados e agrupados para gerar as estatísticas apresentadas neste estudo foram os de domicilio, morador e caderneta de despesas (IBGE, 2004 e 2010).

O consumo de pescados fora do domicilio foi estimado a partir do bloco de consumo alimentar pessoal da POF 2008-2009 (Tabela 1). Neste questionário os indivíduos foram orientados a registrar e relatar detalhadamente os nomes dos alimentos consumidos, o tipo de preparação, a medida usada, a quantidade consumida, o horário e se o consumo do alimento ocorreu no domicilio ou fora dele. Foram pesquisados os moradores com 10 anos ou mais de idade de 13.569 domicílios (24,3\% dos domicílios pesquisados) ou 34.003 moradores (IBGE, 2011). 
Tabela 1. Consumo alimentar médio per capita e percentual de consumo fora do domicílio em relação ao total consumido, por sexo, segundo os alimentos - Brasil período 2008-2009

Percentual de consumo fora do domicílio em relação ao total consumido (\%)

\begin{tabular}{lccc}
\hline Alimentos & Total & Masculino & Feminino \\
\hline Peixes frescos e preparações & 10,8 & 11,5 & 10,1 \\
Peixes em conserva & 9,4 & 9,4 & 9,5 \\
Peixes salgados & 10,6 & 10,5 & 10,7 \\
Outros pescados & 24,2 & 26,6 & 21,3 \\
\hline
\end{tabular}

Fonte: (IBGE, 2011)

Portanto, a demanda por pescados foi definida como:

$$
\begin{gathered}
\mathrm{D}=\mathrm{C}_{\mathrm{e}}+\mathrm{F}+\mathrm{P} \\
\mathrm{C}_{\mathrm{e}}=\mathrm{C} \times \mathrm{f}_{\mathrm{C}} \\
\mathrm{F}=\mathrm{C}_{\mathrm{e}} \times\left(\frac{1}{1-\mathrm{i}}-1\right)
\end{gathered}
$$

Onde, $\mathrm{D}=$ Demanda por Pescados Inteiro $(\mathrm{t}) ; \mathrm{C}_{\mathrm{e}}=$ Consumo Domiciliar Equivalente de Pescado Inteiro $(t) ; f_{C}=$ Fator de correção para equivalente pescado inteiro consumido no domicilio $\left(1<\mathrm{f}_{\mathrm{c}}<4\right) ; \mathrm{F}=$ Consumo Fora do Domicilio $(\mathrm{t})$; $\mathrm{i}=$ Percentual de consumo fora do domicílio em relação ao total consumido; $P=$ Perdas Estimadas (t).

A partir de informações obtidas através de séries históricas de quantidades brutas de produção, importação e exportação de pescados (Abdallah, 1998; IBAMA, 2003, 2004, 2007; MPA, 2012, 2013, 2014); e, de PIB per capita de 1970 à 2013 (IPEADATA, 2014) foi estimada uma equação de consumo aparente em função de renda para verificar a aderência dos dados corrigidos e a tendência obtida.

Buscando um melhor ajuste da função foi aplicada uma transformação monotônica nos dados (logaritmo neperiano). A função foi estimada com dados entre 1970 a 2007, período preliminar ao MPA assumir a elaboração das estatísticas de produção. No período entre 1988 à 1995 foi aplicada uma variável dummy para evitar as distorções causadas pelos subsequentes planos econômicos impostos pelos governos da época. A função pode ser expressa como:

$$
\mathrm{CA}_{70-07}=\alpha+\mathrm{D}_{88-95}+\beta \cdot \mathrm{R}_{70-07}+\mathrm{e}
$$


Onde, $\mathrm{CA}_{70-07}=$ logaritmo neperiano do consumo aparente ${ }^{4} ; \alpha=$ intercepto; $D_{88}$ 95 = variável dummy (1 para 1988 à 1995 e 0 para os demais períodos); $\beta$ = parâmetro; $\mathrm{R}_{70-07}=$ logaritmo neperiano do PIB per capita real deflacionado para janeiro de 2014; e $=$ erro.

\section{Resultados e Discussão}

A produção média estimada para os anos de 2002-2003 foi de, aproximadamente, 999 mil t. Já para os anos de 2008-2009 a produção média foi de 1.212 mil t $^{5}$.

A importação média de pescados apresentou um crescimento de 53\% no período, passando de 143,45 mil t em 2002-03 para 219,57 mil t em 2008-09 ${ }^{6}$. Para o cálculo dos fatores de correção por período dividiu-se o valor médio corrigido pelo valor médio importado, obtendo-se 294,10 mil t em 2002-03* para 499,18 mil t em 2008-09*. Observa-se que além do aumento liquido no volume de importações e no percentual de produtos mais elaborados $\left(f_{i} 2002-03=2,05\right.$ e $\left.f_{i} 2008-09=2,27\right)$.

Analogamente, as exportações médias de pescados no período reduziram $67 \%$ no período (de 110,10 para $33,47 \mathrm{mil} \mathrm{t}^{7}$ ). Utilizaram-se os mesmos critérios para 0 cálculo dos equivalentes pescados inteiros exportados, as exportações seguiram a mesma tendência das importações, de comercialização de produtos mais processados

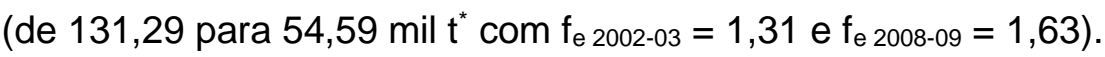

Aplicando as fórmulas de oferta de equivalente pescado inteiro, para os períodos 2002-03 e 2008-09 se obtém:

$$
\begin{gathered}
\mathrm{O}_{2002-03}=998,57+294,10-131,29 \\
\mathrm{O}_{2002-03}=1161,38 \\
\mathrm{O}_{2008-09}=1.198,62+499,18-54,59 \\
\mathrm{O}_{2008-09}=1643,21
\end{gathered}
$$

Embora possa ser notado aumento significativo da oferta entre os períodos analisados, o consumo domiciliar praticamente não se alterou no período, mantendo-se na casa das 770 mil t/ano. Contudo, apesar da quantidade total bruta consumida no

\footnotetext{
${ }^{4}$ Consumo Aparente $=$ Produção + Importação - Exportação (quantidade bruta de pescados em $\mathrm{t}$ )

$5 \frac{\mathrm{O}_{2002}+\mathrm{O}_{2003}}{2}=\frac{1.006,88+990,27}{2}$ e $\frac{\mathrm{O}_{2008}+\mathrm{O}_{2009}}{2}=\frac{1.156,42+1.240,81}{2}$

$6 \frac{\mathrm{I}_{2002}+\mathrm{I}_{2003}}{2}=\frac{286,90}{2}$ e $\frac{\mathrm{I}_{2008}+\mathrm{I}_{2009}}{2}=\frac{439,14}{2}$

$7 \frac{E_{2002}+E_{2003}}{2}=\frac{200,19}{2}$ e $\frac{E_{2008}+E_{2009}}{2}=\frac{66,95}{2}$
} 
domicilio permanecer praticamente constante, o padrão de consumo se alterou ligeiramente, sendo que a população tem optado por produtos mais processados (de

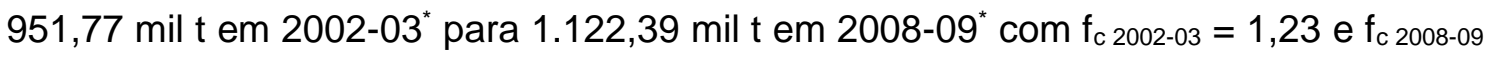
$=1,45)$.

Para calcular o consumo de pescados fora do domicilio, utilizam-se os padrões obtidos no bloco de consumo alimentar pessoal da POF 2008-2009 e extrapolados para as informações da POF 2002-2003. Esta aproximação foi necessária pelo fato da informação do bloco de consumo alimentar pessoal da POF 2002-2003 não estar disponível no mesmo formato e justificado pelo fato das quantidades consumidas pelos domicílios em ambas as pesquisas apresentarem magnitudes semelhantes.

O cálculo indica um consumo fora do domicilio de equivalente peixe inteiro de 114,36 mil t em 2002-2003 e de 142,17 mil t para 2008-2009, ou seja, mantendo-se o mesmo percentual de consumo fora do domicílio, o aumento do equivalente peixe inteiro ocorre em função da alteração no padrão de consumo deste produto (maior demanda por produtos mais processados). As informações do Infopesca de 2009 [Neiva et.al. (2010); Barroso e Wiefels (2010); Borges (2010); Gandra (2010)] indicam que o consumo médio de equivalente pescado inteiro fora do domicilio nas quatro regiões metropolitanas estudadas, em 2010, poderia chegar a $40 \%$.

Igualando-se a oferta e a demanda de pescados, por diferença, obtém-se uma estimativa das perdas:

$$
\begin{gathered}
\mathrm{O}_{2002-03}=\mathrm{D}_{2002-03} \\
1161,38=951,77+114,36+\mathrm{Pe}_{2002-03} \\
\mathrm{Pe}_{2002-03}=95,25 \\
\mathrm{O}_{2008-09}=\mathrm{D}_{2008-09} \\
1643,21=1122,39+142,17+\mathrm{Pe}_{2008-09} \\
\mathrm{Pe}_{2008-09}=378,65
\end{gathered}
$$

Embora a variável de perdas possa ter absorvido todos os possíveis erros do equilíbrio imposto, não é aceitável que este número cresça de $8,20 \%$ para 23,04\% sobre a oferta em seis anos.

De forma prudente, mantendo-se o mesmo percentual de perdas de 2002-2003 para 2008-2009:

$$
\begin{gathered}
\mathrm{O}_{2008-09}=\mathrm{D}_{2008-09} \\
\mathrm{O}_{2008-09}=1122,39+142,17+\mathrm{O}_{2008-09} \times 8,20 \% \\
\mathrm{O}_{2008-09}=1377,52
\end{gathered}
$$


Ou seja, a oferta de pescados em 2008-2009 reduziria de 1643,21 mil t para 1264,56 mil t (desconsiderando-se as perdas) ou para 1377,52 mil t (considerando-se $8,20 \%$ de perdas).

Se as estatísticas de demanda e comercio internacional (importação e exportação) estiverem corretas, resta uma análise mais apurada dos dados de produção de pescados do Brasil. Entre os anos de 2002-2003 e os anos 2008-2009 a produção pesqueira oficial apresentou um crescimento de $20 \%$, ou 200,04 mil t. Considerando a oferta corrigida de pescados na ordem de 1377,52 mil t, há um déficit de 265,69 mil t entre a estatística oficial e a corrigida. Portanto existem fortes indícios que a produção de pescados brasileira entre os anos de 2002-2003 e os anos 2008-2009 teve um decréscimo de 65,65 mil t (déficit 6,90\% ${ }^{8}$ em relação à produção de pescados de 2002 2003). Ao se considerar as perdas como erro, o déficit pode ser chegar a $9,23 \%{ }^{9} \mathrm{em}$ relação à produção de pescados de 2002-2003 corrigida de 903,32 mil t.

Considerando a que a estimativa da população brasileira era de 175,85 milhões e de 190,52 milhões de habitantes em 2003 e 2009 respectivamente, enquanto a estatística oficial aponta um crescimento no consumo per capita aparente de 6,60 para $8,62 \mathrm{~kg} / \mathrm{hab}$.ano. Os dados corrigidos apresentam um crescimento mais modesto que varia de 6,60 para 7,23 kg/hab.ano a 6,06 para 6,64 kg/hab.ano (Tabela 2).

\footnotetext{
*significativo à $1 \%$

$865,65 / 998,57$

${ }^{9}[(998,57-95,25)-(1198,62-378,65)] /(998,57-95,25)$
} 
Tabela 2. Análise dos cenários: dados Oficiais, considerando o mesmo percentual de Perdas (Pe) de 2002-2003 para 2008-2009 e desconsiderando as perdas para Oferta, Demanda (mil t) e Consumo Per Capita (CP em kg/hab.ano) de Pescados no Brasil em 2002-2003 e em 2008-2009.

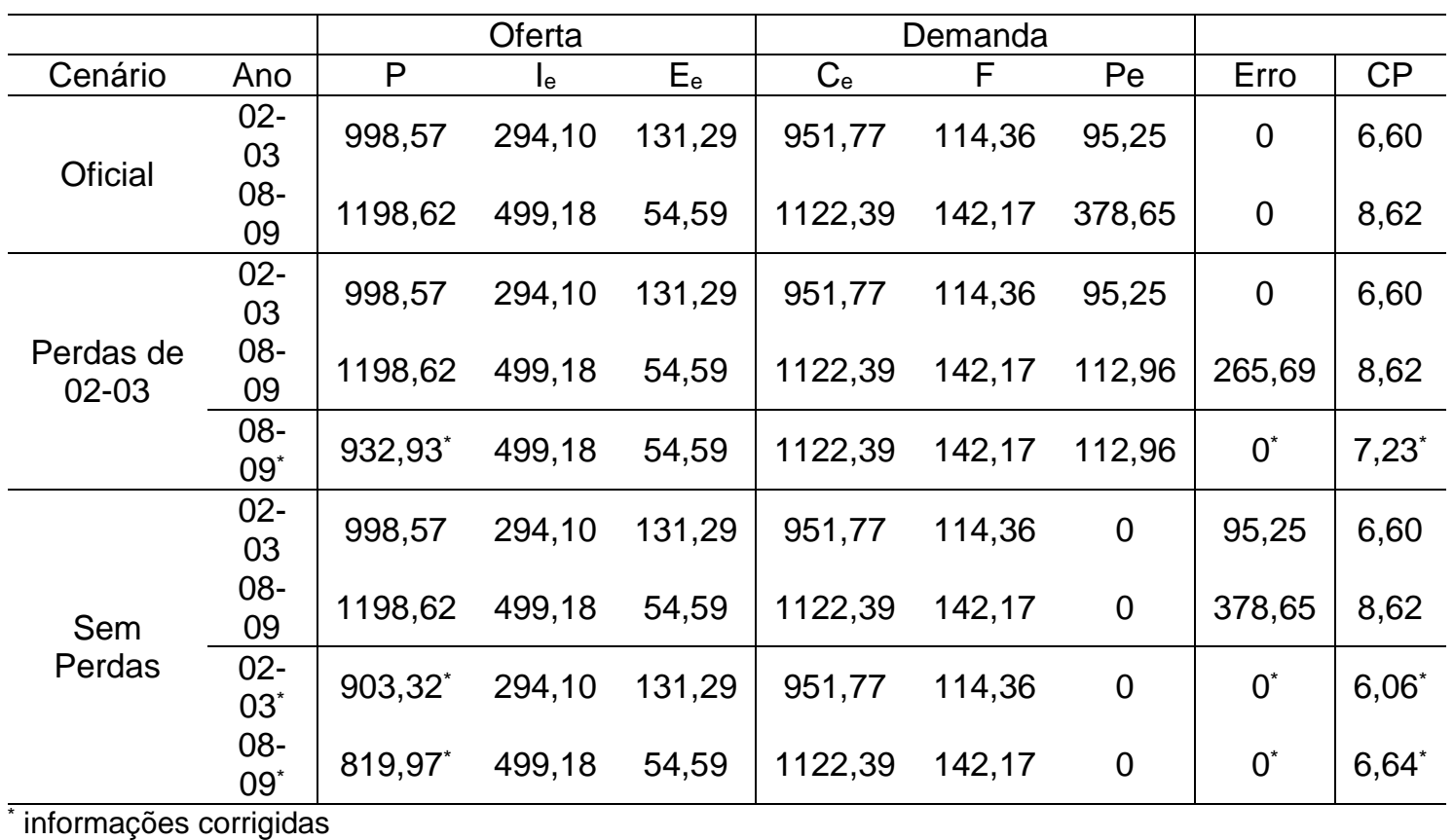

Para verificação da aderência dos dados corrigidos pela POF e com a tendência dos anos anteriores obteve-se a seguinte função:

$$
C A=4,745899^{*}-0,15504^{*} \cdot D_{88-95}+0,921724^{*} \cdot R_{70-07}+e
$$

O teste $F=137,60^{*}$, com $R$ múltiplo $=0,9418$ e $R^{2}=0,8871$. A Figura 1. ilustra a aderência dos dados à função estimada. Dados da FAO (2014) trazem estimativas de elasticidades de renda para consumo de pescados no Brasil ainda mais conservadoras, 0,85 e alternativamente 0,571 utilizada para suas projeções. Este estudo também aponta uma queda na produção aquícola brasileira de 701 mil t para 629 mil t entre 2010 e 2011. 


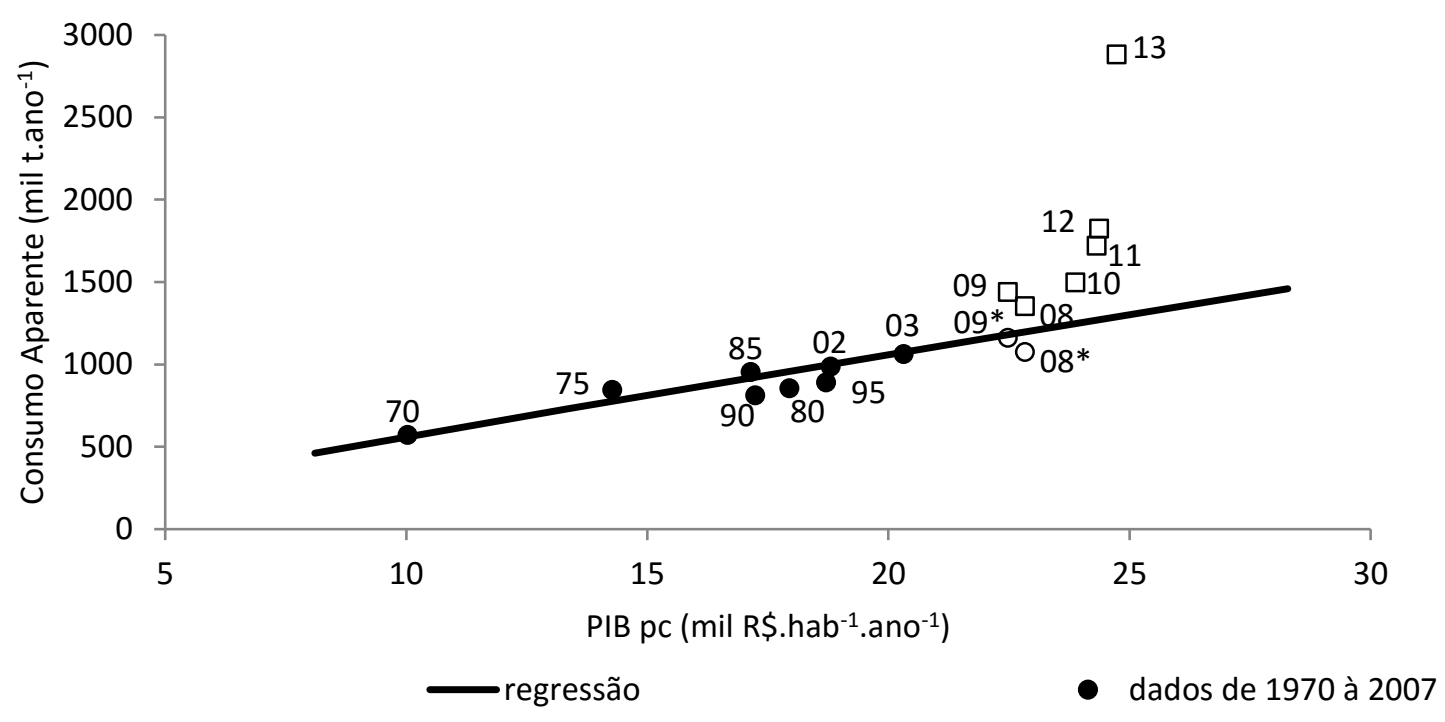

Figura 1. Consumo aparente de pescados em mil t.ano-1 ${ }^{-1}$ em função do PIB per capita em R \$ mil.(hab/ano) no Brasil de 1970 a 2013.

Fonte: Abdallah, 1998; IBAMA, 2003, 2004, 2007; MPA, 2012, 2013, 2014; IPEADATA, 2014; IBGE, 2004; e IBGE, 2011.

Ao considerarmos a metodologia que considera perdas nos dados da POF, os consumos aparentes dos anos de 2002, 2003 e 2009 estão dentro do erro padrão da função estimada e o dado de 2008 está abaixo. Por outro lado, o consumo aparente obtido através dos dados oficiais de produção de 2008 a 2013 estão consistentemente acima do erro padrão da função estimada. Esta análise reforçada pelos dados de produção de aquicultura de 2013 levantados por IBGE (2014) de 476,5 mil t, 32,6\% inferior à produção apontada pelo MPA em 2012 (707,4 mil t) e superior à informação de 2009 (415,6 mil t), indicam que os dados oficiais da produção de pescados estão superestimados. A função estimada reforça a hipótese de que os dados de consumo aparente corrigidos pela POF 08-09 sigam a mesma tendência dos dados de 1970 a 2007.

\section{Conclusão}

Notou-se a tendência de aquisição de pescados com maior grau de processamento pelos consumidores. Enquanto os dados oficiais apontam um crescimento do consumo per capita aparente de $2 \mathrm{~kg} / \mathrm{hab}$.ano, os dados corrigidos apresentam um crescimento mais modesto (em torno de 0,6 kg/hab.ano) entre os anos de 2002-2003 e 2008-2009. Ao se considerar as estatísticas de demanda e comercio internacional (importação e exportação) como correta, enquanto os dados oficiais de 
produção de pescados apontam um crescimento na ordem de $20 \%$, os dados corrigidos apontam um decréscimo de 6,9\% e 9,2\% entre os anos de $2002-2003$ e 2008-2009. As informações geradas a partir dos dados oficiais de 2008 a 2013 estão consistentemente acima do erro padrão de regressão, o que indica que os dados oficiais de produção de pescados estão superestimados.

\section{Referências}

Abdallah, P.R. 1998. Atividade pesqueira no Brasil: política e evolução. Tese (Doutorado) - Escola Superior de Agricultura "Luiz de Queiroz", Universidade de São Paulo. Piracicaba. 137p.

Barroso, R.M.; Wiefels, A.C. 2010. O mercado de pescado da região metropolitana do Rio de Janeiro 2010. Proyecto Mejoramiento Del Acesso a los Mercados de Productos Pesqueros y Acuícolas de La Amazonia. CFC/FAO/INFOPESCA. 104 p.

Borges, A.M. 2010. O mercado do pescado em Brasília. Proyecto Mejoramiento Del Acesso a los Mercados de Productos Pesqueros y Acuícolas de La Amazonia. CFC/FAO/INFOPESCA. 109 p.

Food and Agriculture Organization [FAO]. 2014. Economic analysis of supply and demand for food up 2030 - special focus on fish and fishery products. Fishery and Aquiculture Circular. n. 1089. 106 p.

Gandra, A.L. 2010. O mercado do pescado da região metropolitana de Manaus 2010. Proyecto Mejoramiento Del Acesso a los Mercados de Productos Pesqueros y Acuícolas de La Amazonia. CFC/FAO/INFOPESCA. 84p.

Instituto Brasileiro de Geografia e Estatística [IBGE]. 2004. Pesquisa de orçamentos familiares 2002-2003: microdados. 1 CD-ROM.

Instituto Brasileiro de Geografia e Estatística [IBGE]. 2011. Disponível em: <http://www.ibge.gov.br/home/estatistica/populacao/condicaodevida/pof/2008_2009/mi crodados.shtm>. Acesso em: 12 out. 2011.

Instituto Brasileiro de Geografia e Estatística [IBGE]. 2010. Pesquisa de Orçamentos Familiares 2008-2009 - Despesas, Rendimentos e Condições de Vida. Rio de Janeiro. $222 p$.

Instituto Brasileiro de Geografia e Estatística [IBGE]. 2011. Pesquisa de Orçamentos Familiares 2008-2009 - Análise do consumo alimentar pessoal no Brasil. 150p.

Instituto Brasileiro do Meio Ambiente e dos Recursos Naturais Renováveis [IBAMA]. 2003. Estatística da pesca 2001 Brasil: grandes regiões e unidades da federação. $124 \mathrm{p}$.

Instituto Brasileiro do Meio Ambiente e dos Recursos Naturais Renováveis [IBAMA]. 2004. Estatística da pesca 2003 Brasil: grandes regiões e unidades da federação. 137 p. 
Instituto Brasileiro do Meio Ambiente e dos Recursos Naturais Renováveis [IBAMA]. 2007. Estatística da pesca 2005 Brasil: grandes regiões e unidades da federação. 147 p.

Instituto Brasileiro do Meio Ambiente e dos Recursos Naturais Renováveis [IBAMA]. 2007. Estatística da pesca 2007 Brasil, grandes regiões e unidades da federação. IBAMA/DBFLO/CGFAP. 113 p.

Instituto de Pesquisas de Economia Aplicada [IPEADATA]. 2014. Disponível em:<http://www.ipeadata.gov.br/>. Acesso em 22 ago. 2014.

Ministério do Desenvolvimento, Indústria e Comercio Exterior [MDIC]. 2012.

Aliceweb2. Disponível em: < http://aliceweb2.mdic.gov.br/>. Acesso em 25 mai. 2012.

Ministério da Pesca e Aquicultura [MPA]. 2012. Boletim estatístico da pesca e aquicultura - Brasil 2010. Disponível em: <http://www.mpa.gov.br/informacoes-eestatisticas/estatistica-da-pesca-e-aquicultura>. Acesso em: Acesso em 25 mai. 2012.

Ministério da Pesca e Aquicultura [MPA]. 2013. Boletim estatístico da pesca e aquicultura - Brasil 2011. Disponível em: <http://www.mpa.gov.br/images/Docs/Informacoes_e Estatisticas/Boletim\%20MPA\%202011FINAL2.pdf>. Acesso em: Acesso em 23 setembro 2013.

Ministério da Pesca e Aquicultura [MPA]. 2014. Balanço 2013 - pesca e aquicultura. Disponível em: <http://www.mpa.gov.br/images/Docs/Publicidade/CartilhaBalan\%C3\%A7o-2013Minist\%C3\%A9rio-Pesca-Aquicultura.pdf> Acesso em: Acesso em 25 set. 2014.

Ministério da Pesca e Aquicultura [MPA]. 2014. 1․ Anuário brasileiro da pesca e aquicultura - Brasil 2014. Disponível em: <http://issuu.com/revistas_nd/docs/anu_rio_ pesca_e_aquicultura_2014_i >. Acesso em: Acesso em 03 ago. 2014.

Neiva, C.R.P.; Tomita, R.Y; Cerqueira, M.A.S.; Miura, M.; Furlan, E.F.; Machado; T.M.; Lemos Neto, M.J. 2010. Estudo: "O mercado de pescado da região metropolitana de São Paulo". Proyecto Mejoramiento Del Acesso a los Mercados de Productos Pesqueros y Acuícolas de La Amazonia. CFC/FAO/INFOPESCA. 86 p.

Sidonio, L., Cavalcanti, I., Capanema, L. Morch, R., Magalhães, G. Lima, J., Burns, V., Alves Sr., A. E Mungioli, R. 2012. "Panorama da aquicultura no Brasil: desafios e oportunidades". BNDES Setorial 35, p. 421-463. 2012. Disponível em: <http://www.bndes.gov.br/SiteBNDES/export/sites/default/bndes_pt/Galerias/ Arquivos/conhecimento/bnset/set3512.pdf> Acesso em 20 de ago. 2012. 